\title{
Vaccination by COVID-I 9 in the elderly population and its relationship with life expectancy
}

\begin{abstract}
Excess mortality during the coronavirus pandemic in the world is one of the phenomena that could most impact demographic indicators such as life expectancy. A short review was made of why this happens and what could counter it if there is a solution, from which, I conclude that it could be a judicious vaccination not only in the elderly population but also in people with associated comorbidities.
\end{abstract}

Keywords: mortality, life expectancy, covid-19, elderly population

\author{
Volume 6 Issue 3 - 202 I
}

\author{
Juan de Jesús Sandoval \\ Research Scientist, Independent Consultant in Demography and \\ Statistics, Medellín, Colombia
}

Correspondence: Juan de Jesús Sandoval, Research Scientist, Independent Consultant in Demography and Statistics, Medellín, Colombia, Email juan.sandoval2@gmail.com

Received: March 24, 2021 | Published: May 20, 2021

\section{Introduction}

According to the latest data from the Worldometer, 2021, ${ }^{1}$ as of March 12, 2021, there were 2,641,683 deaths from COVID-19 in the world, with an mortality rate mean of 338.9 deaths per million inhabitants. However, there were countries like Belgium and the United Kingdom with rates of 1,922 and 1,837 respectively. On the other side, the lowest rates in the world, countries like South Korea with 32 or Australia with 35 deaths per million inhabitants. As stated by the Centers for Disease Control and Prevention (CDC), in the United States, during the pandemic, $81 \%$ of all deaths from COVID-19 had occurred in the age group over 64 and 59\% in those over 74 . In addition, the $55 \%$ of deaths occurred in men (National Center for Health Statistics, 2021). ${ }^{2}$

In conformity with the above and Bhopal \& Bhopal, ${ }^{3}$ age and sex are associated with mortality from COVID-19. Similarly, in research by Yáñez et al, ${ }^{4} 2020$ in 16 countries, they showed that compared to people aged 54 years or younger, the incident rate ratio (IRR) was 8.1, "indicating that the mortality rate of COVID-19 was 8.1 times higher $(95 \% \mathrm{CI}=7.7,8.5)$ among those 55 to 64 years, and more than 62 times higher $(\mathrm{IRR}=62.1 ; 95 \% \mathrm{CI}=59.7,64.7)$ among those ages 65 or older. Mortality rates from COVID- 19 were $77 \%$ higher in men than in women $(\mathrm{IRR}=1.77,95 \% \mathrm{CI}=1.74,1.79) "{ }^{4}{ }^{4}$

An important indicator in the mortality evolution, useful for demographers, is life expectancy. This represents the number of years that, on mean, a person of a generation would live subject to a mortality experience represented in a life table, if their mortality conditions were kept constant throughout life. $^{5}$ Therefore, it is important to highlight that life expectancy is strongly influenced by the extremes of the mortality curve, that is, by child mortality or adult mortality. In such a way that, with the increasing number of centenarians and supercentenarians in the world, ${ }^{6,7}$ the life expectancy of people has been increasing in all countries.

However, with the COVID-19 pandemic where, according to the information presented above, mortality from cases of this disease has mainly affected the elderly population and of them, in a greater proportion at men. This shows in some way that "the age patterns of COVID-19 mortality are such that vaccinating the oldest first saves the most lives and, surprisingly, also maximizes years of remaining life expectancy". ${ }^{9}$
The same authors also expressed that it will be necessary to appeal to common intuition, to achieve a balance between saving the greatest number of lives and being able to balance more life expectancy in the future. To do this, they expressed that it is easier to put aside the fears of, to whom to direct vaccination as a priority and to give priority to vaccines in the elderly population and the most vulnerable health cases of the population. Otherwise, there would be a negative impact on life expectancy due to COVID-19 in the world.

In other words, priority must be given to vaccinating older adults to reduce the negative impact of COVID-19 on life expectancy and, therefore, public policies for them and various indicators that depend of life expectancy, in that case, should be reevaluated. The same authors showed that for the case of COVID-19, the general properties of the life table can be invoked to remove the potential conflict between saving lives and maximizing future life years. And therefore, administer COVID-19 vaccines to those who face a greater risk of death from the disease and thus be able to achieve the objectives of reducing mortality and its low impact on life expectancy.

\section{Conclusion}

Based on this review, several things can be concluded. The first, without a doubt, vaccination will save more lives and therefore allow a less impact on life expectancy in the years of life lost. Second, vaccination should be prioritized not only for older adults, but also for those with chronic disease comorbidities associated with COVID-19, since both groups are highly vulnerable. The empirical analysis by Goldstein et al, 2021 showed that it is easier than previously thought to put those fears aside and prioritize vaccines for the elderly and those in the most vulnerable states of health. Where, according to their results, they concluded that vaccination of people with poorer health at a certain age can save more years of life than vaccination of healthy people of the same age.

Finally, it is essential to note that the increased risk of mortality from COVID-19 increases with age, but also the beliefs or myths about not being vaccinated. Therefore, it is important to dispel fears of non-vaccination in older ages with education and reinforcement policies in vaccination campaigns.

\section{Funding}

None. 


\section{Acknowledgments}

None.

\section{Conflicts of interest}

The author declares that there is no conflict of interest about the publication of this paper.

\section{References}

1. Worldometer. COVID-19 Coronavirus Pandemic. Coronavirus Pandemic. 2021.

2. National center for health statistics. Centers for disease control and prevention. Weekly updates by select demographic and geographic characteristics. 2021.

3. Bhopal Yanez ND, Weiss NS, Romand JA, et al. COVID-19 mortality risk for older men and women. BMC Public Health. 2020;20(1):1742.

4. Preston SH, Heuveline P, Guillot M. Demography: measuring and modeling population processes. Pop Dev Rev. 2001;27:365.
5. Nepomuceno MR, Turra CM. The population of centenarians in brazil: historical estimates from 1900 to 2000. Population and Development Review. 2020;46(4):813-833.

6. Rau R, Soroko E, Jasilionis D, et al. Continued reductions in mortality at advanced ages. Population and Development Review. 2008;34(4):747768.

7. Bhopal SS, Bhopal R. Sex differential in COVID-19 mortality varies markedly by age. In The Lancet. 2020;396(10250):532-533.

8. Andrasfay T, Goldman N. Reductions in 2020 US life expectancy due to COVID-19 and the disproportionate impact on the Black and Latino populations. Proceedings of the National Academy of Sciences. 2021;118(5).

9. Goldstein, JR., Cassidy T, Wachter KW. Vaccinating the oldest against COVID-19 saves both the most lives and most years of life. Proceedings of the National Academy of Sciences of the United States of America. 2021;118(11):11-13. 